\title{
Mapping evidence on decision-making on contraceptive use among adolescents: a scoping review protocol
}

\author{
Mumbi Chola ${ }^{1,2^{*}}$, Khumbulani Hlongwana ${ }^{1}$ and Themba G. Ginindza ${ }^{1}$
}

\begin{abstract}
Background: Contraceptive use among adolescents remains consistently low globally. Numerous studies have been done investigating factors that contribute to low contraceptive prevalence rates in this special population. It is particularly vital to understand decision-making processes that adolescents undergo when deciding whether or not to use contraceptives. Therefore, this scoping review seeks to map available evidence on decision-making processes in contraceptive use among adolescents.

Methods: We will conduct a scoping review to explore, describe and map literature on the adolescent decisionmaking regarding contraceptive use. The primary search will include peer-reviewed and review articles. Databases, including PubMed, MEDLINE with Full Text via EBSCOhost, PsychINFO via EBSCOhost, CINAHL with Full Text via EBSCOhost, Google Scholar, Science Direct and Scopus, will be searched for articles that meet the eligibility criteria. Keyword searches will be used, and for articles included after title screening, abstract and full articles will be screened by two independent reviewers with a third as a decider on any disputes. Content analysis will be used to present the narrative account of the reviews.

Discussion: Understanding how adolescents make decisions about whether or not to use contraception is essential for improving contraceptive prevalence rates in this special population. It is envisioned that the results from this review will highlight key evidence on how adolescent make decisions regarding contraceptive use as well as gaps and opportunities for future research. It will also be important in enhancing and re-focusing adolescent sexual and reproductive health policies and programmes.
\end{abstract}

Keywords: Adolescents, Decision making, Contraception, Contraceptive use

\section{Background}

Modern contraceptive methods are products or medical procedures that interfere with reproduction from acts of sexual intercourse [1]. Types of modern contraceptives include sterilisation (male and female), intrauterine devices and systems, subdermal implants, oral contraceptives, condoms (male and female), injectables, emergency contraceptive pills, patches, diaphragms and cervical caps, spermicidal agents (gels, foams, creams, suppositories, etc.), vaginal rings and sponge [1].

\footnotetext{
* Correspondence: mumbi24@gmail.com

'Discipline of Public Health Medicine, School of Nursing and Public Health, University of KwaZulu-Natal, Durban 4041, South Africa

2Department of Epidemiology \& Biostatistics, School of Public Health,

University of Zambia, Lusaka, Zambia

Globally, the prevalence of contraceptive use varies. In 2015 , two out of three women or about $64 \%$ of women of reproductive age (15-49), married or in a union, were using some form of contraception, either modern or traditional [2]. Eastern and Southern Africa and West and Central Africa recorded lower numbers with $38.6 \%$ and $17.6 \%$ respectively [2]. Statistics are even lower among adolescents [3-5] with only about $15 \%$ of girls in developing countries aged 15-19, married or in a union, using modern contraceptive methods [6]. Adolescence is defined as all persons aged 10 to 19 years [7], and it is further subdivided into early adolescence (11 to 13 years), adolescence (14-17 years) and young adulthood (18-25 years), which encompasses those aged 18-19 years [8]. 
Low contraception use in this age group exposes adolescents to higher risks of maternal mortality, obstructed labour and obstetric fistula, and results in lower chances of receiving an education and obtaining employment [9-11]. Children born to adolescent mothers also face higher risks of mortality, undernourishment and school dropout compared to their peers [12].

While factors that contribute to low contraceptive use among adolescents have been well documented, it is particularly vital to understand decision-making process they undergo when deciding on whether or not to use contraceptives. This information will be vital for policy-makers and program managers in addressing poor contraception usage among adolescents as well as preventing maternal complications. It will also be important in enhancing and re-focusing adolescent sexual and reproductive health policies and programmes.

Therefore, this scoping review seeks to:

- Map existing literature on adolescent decisionmaking on contraceptive use in sub-Saharan Africa

- Map existing literature on the influence of parental, societal and peer-related factors on adolescents' decision to use contraception

Findings from this review will highlight gaps in literature and form the basis for refining research questions for further research.

\section{Methods}

\section{Scoping review}

This is a scoping review of literature on adolescents' decision-making on contraceptive use. This review is part of a larger study whose aim is to examine the levels, patterns and trends of contraception use among adolescents and understand their decision-making, as well as their needs, preferences and perspectives regarding existing and future contraceptive methods. The review has been written using the PRISMA-P [13] as a guide and will be based on the methodological framework for scoping studies as proposed by Arksey and O'Malley's [14]. The framework stipulates the following steps:

- Identifying the research question

- Identifying relevant studies

- Study selection

- Charting the data

- Collating, summarising and reporting the results

This scoping review will, however, include a quality appraisal of the studies included in the review as proposed by Levac et al. [15].

\section{Identifying the research question}

What is the available evidence on decision-making in contraceptive use among adolescents?

Sub-questions include:

1. What societal and peer factors influence adolescents' decision-making on contraceptive use?

2. What parental factors influence adolescents' decision-making on contraceptive use?

3. What individual factors influence adolescents' decision-making on contraceptive use?

\section{Eligibility criteria}

Eligibility criteria will be based on the following inclusion and exclusion criteria.

\section{Inclusion criteria}

Studies present evidence on:

- Adolescent boys and girls aged 10-19 years

- Decision-making in contraceptive use among adolescents aged 10-19 years

- Parental influences on adolescents' decision to use contraceptives

- Societal and peer influences on adolescents' decision to use contraceptives

- Individual or "self" influence on adolescents' decision to use contraceptives

- Published studies including guidelines, reports, technical or policy briefs and opinion papers and other grey literature

\section{Exclusion criteria}

Studies meeting the following criteria will be excluded:

- Studies with no evidence on decision-making in contraceptive use among adolescents

- Studies with no evidence on influence of parental, societal, peer or individual factors on decisionmaking in contraceptive use among adolescents

- Studies not focused on adolescents aged 10-19 years

- Studies not freely available in full text

\section{Eligibility of research question}

The study has used the Population-Concept-Context (PCC) framework (see Table 1) recommended by the Joanna Briggs Institute for scoping reviews [16] to determine the eligibility of research question. This is a more flexible alternative to the PICO (Population, Intervention, Comparator and Outcome) framework recommended for systematic reviews. 
Table 1 PCC framework

\begin{tabular}{ll}
\hline Population & Adolescents aged 10-19years. This is based on the World Health Organization (WHO)'s definition of adolescents [21]. \\
Concept & $\begin{array}{l}\text { Decision-making in contraceptive use. This includes factors that adolescents consider and processes that they go through in } \\
\text { deciding whether or not to use contraceptives }\end{array}$ \\
Context & Global-including studies from high-income and LMICs
\end{tabular}

Identifying relevant studies (search strategy)

The following databases will be searched for articles that meet the eligibility criteria. These are PubMed, MEDLINE with Full Text via EBSCOhost, PsychINFO via EBSCOhost, CINAHL with Full Text via EBSCOhost, Google Scholar, Science Direct and Scopus. Search will follow the PRISMA guidelines

\section{Identifying relevant studies (search strategy)}

The following databases will be searched for articles that meet the eligibility criteria. These are PubMed, MEDLINE with Full Text via EBSCOhost, PsychINFO via EBSCOhost, CINAHL with Full Text via EBSCOhost, Google Scholar, Science Direct and Scopus. Search will follow the PRISMA guidelines.

Articles will also be searched through the 'Cited by' search and reference lists of included articles. Search strategy was piloted to check the appropriateness of selected electronic databases and key words (Table 2). Boolean terms AND and OR will be used to separate the keywords during the search. Mesh terms (Medical Subject Headings) will also be included in the search.

A library will be created for this review using EndNote $\times$ 8.0.2 referencing software. The primary investigator will conduct a comprehensive search and screening of the study titles from the abovementioned databases. All studies with eligible titles will be exported to the EndNote library, and all duplicates will be removed before abstract screening. Two reviewers will independently conduct abstract screening followed by full-article screening of selected studies, using standardised tools, with guidance from the eligibility criteria. Where disputes arise, a third reviewer will decide. To optimise the article search strategy, we will utilise our local library services, the UKZN library services, to help with retrieving and finding articles to be included in the full-article screening. Where articles are unavailable, authors will also be contacted. Reporting on these will be done using the Preferred Reporting Items for Systematic Reviews and Meta-Analyses (PRISMA) chart [17], shown in Fig. 1.

\section{Data extraction/charting}

A data charting table (Table 3) will be used to extract background information and process the information from each utilised study. The data extraction form will be developed, piloted and used to extract and process relevant information from each study included. All variables that will focus on answering the research question will be included. The research team will independently conduct a trial data extraction and later discuss as a group to determine consistency of the data extraction approach with the research question and objective. The data extraction form will be continually reviewed and updated in an iterative process. This will improve the quality applicability and consistency of the chart. Once this is completed, the primary author will conduct the data extraction which will be reviewed by the other authors. Any and all discrepancies will be discussed and agreed upon in the final interpretation. All articles reviewed will be assigned a unique code to help track all articles reviewed and those that will be excluded during the data charting process.

\section{Collating, summarising and reporting the results}

Once the data extraction is completed, a narrative account of the data extracted from the included studies will be analysed using the thematic content analysis. Data relating to adolescents' decisions making in contraception and contraceptive use that will be extracted will

Table 2 Keyword searches

\begin{tabular}{|c|c|c|c|}
\hline Date searched & Keyword search terms & Search engine used & Number of studies \\
\hline $21-06-2018$ & $\begin{array}{l}\text { (("adolescent" [MeSH Terms] OR "adolescent" [All Fields]) AND ("decision making" } \\
\text { [MeSH Terms] OR ("decision" [All Fields] AND "making" [All Fields]) OR "decision making" } \\
\text { [All Fields])) AND ("contraception" [MeSH Terms] OR "contraception" [All Fields]) }\end{array}$ & PubMed & 871 \\
\hline \multirow[t]{4}{*}{$21-06-2018$} & \multirow[t]{4}{*}{ Adolescent AND decision making AND Contraception } & Via EBSCOhost & 1752 \\
\hline & & - MEDLINE with Full Text & 653 \\
\hline & & - PsychINFO & 211 \\
\hline & & - CINAHL with Full Text & 108 \\
\hline $21-06-2018$ & Adolescent AND decision making AND Contraception & Google Scholar & 66,000 \\
\hline $21-06-2018$ & $\begin{array}{l}\text { adolescent AND decision AND making AND contraception AND } \\
\text { (LIMIT-TO (ACCESSTYPE (OA)) OR LIMIT-TO (ACCESSTYPE (OTHER))) }\end{array}$ & Scopus & 4836 \\
\hline
\end{tabular}




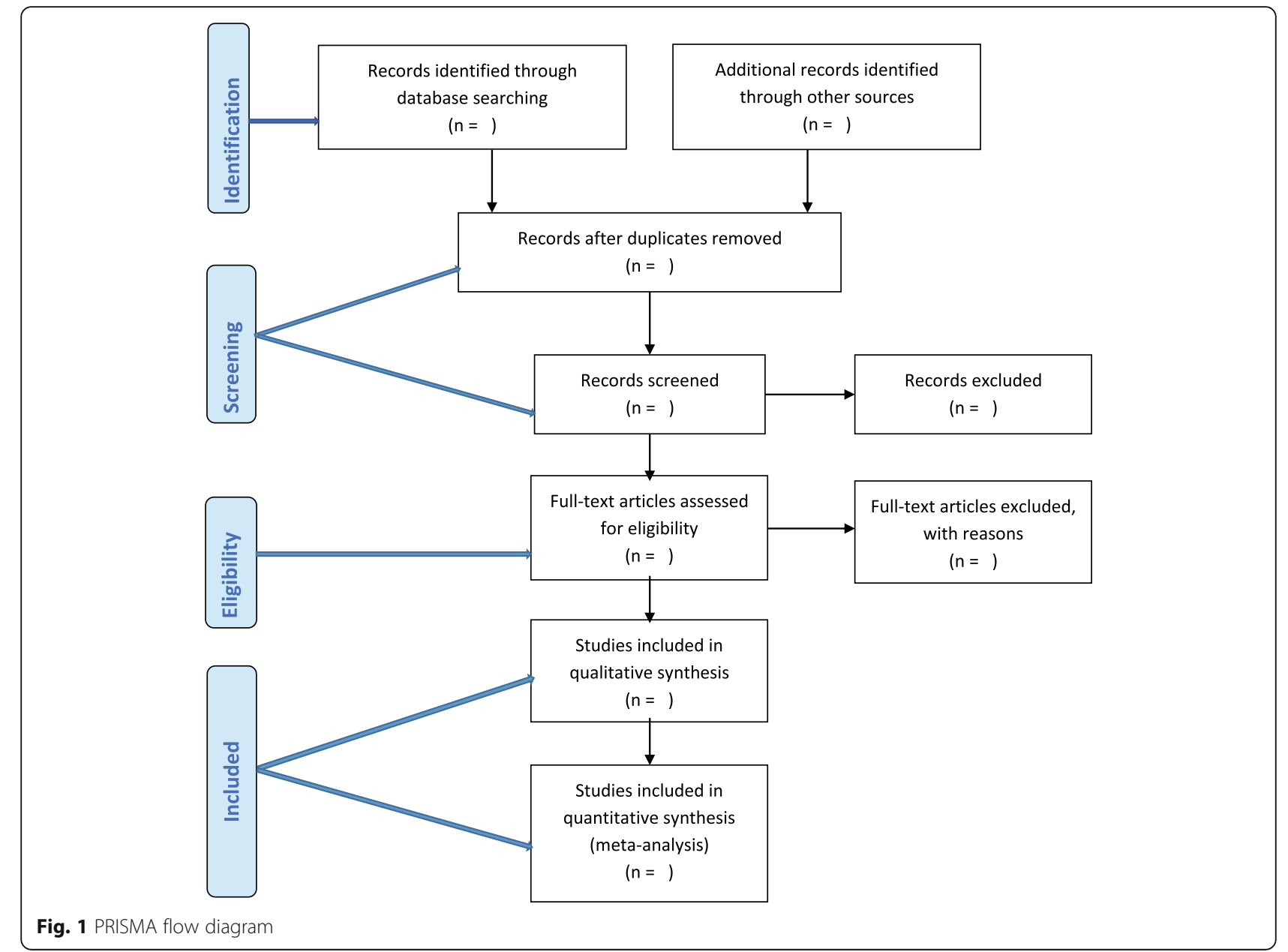

be coded. NVIVO software version 10 [18] will be used collectively to code the data from the included studies. Emerging themes will be identified and data will be coded according to these themes. In line with the general aim of a scoping review, to map out the research landscape, some form of visual representation of the data will be presented in the results section to map the extent, range and nature of research in this area. This will help to identify patterns and themes and postulate explanations for summarising and synthesis of findings. The process will be done as follows [19]:

- Coding data from the included articles

- Categorising the codes into major themes

- Displaying the data

- Identifying key patterns in the data and identify subthemes

- Summarising and synthesising

Resulting themes will then be analysed and synthesised, and their relationship to the research question and objective will be critically examined. The meanings of the findings in relation to the aim of the study and the implications of these findings for future research, policy and practice will be examined.

\section{Quality appraisal}

The Mixed Method Appraisal Tool (MMAT)-version 2011 [20] will be used to determine quality of the studies. Depending on the study design, the appropriate section will be used. Section 1 will be used to appraise qualitative studies; sections 2 to 4 for quantitative studies and section 5 for mixed methods studies. The MMAT will be used to examine the appropriateness of the aim of study, adequacy of methodology, study design, data collection, study selection, data analysis, presentation of findings, author's discussions and conclusions. The scoring matrix in the tool will be used to grade the overall quality. The results from scoring of the abovementioned aspects will determine the quality of resultant article.

\section{Discussion}

Understanding how adolescents make decisions about whether or not to use contraception is essential for 
Table 3 Data extraction form

\begin{tabular}{l} 
Author and date \\
Study title \\
Study population \\
Gender \\
Marital status \\
Education level \\
Methodology \\
Geographical setting (country) \\
Study site \\
Study type \\
Residence \\
Study design \\
Data collection methods \\
Data collection tools \\
Data collection method \\
Sampling \\
Sampling method \\
Intervention (contraception) \\
Contraceptive method \\
Decision-making \\
Data analysis \\
Data analysis type \\
Most important finding limitations and recommendations \\
\hline
\end{tabular}

improving contraceptive prevalence rates in this special population. Increasing contraception among adolescents is important because it will help prevent adverse health such as maternal mortality, obstructed labour and obstetric fistula, and socio-economic outcomes such as diminished opportunities for education and employment [9-11]. Conducting this systematic scoping review will map and document existing evidence on factors that adolescents consider and decision-making processes they go through in deciding whether or not to use contraceptives. This information is vital for understanding why contraceptive prevalence rates among adolescents remain consistently low.

This systematic scoping review will focus on studies published between 1990 and 2017. This is because during this period, there have been various programmes and projects aimed at improving contraception among adolescents in Zambia. The focus is on adolescents because they have been identified as a special population whose health needs have to be prioritised. It is envisioned that the results from this review will highlight key evidence on how adolescent make decisions regarding contraceptive use as well as gaps and opportunities for future research.

\begin{abstract}
Abbreviations
LMIC: Low-to-middle-income country; MMAT: Mixed Method Appraisal Tool; PCC: Population-Concept-Context; PICO: Population, Intervention, Comparator and Outcome; PRISMA: Preferred Reporting Items for Systematic Reviews and Meta-Analyses; UKZN: University of KwaZulu-Natal; WHO: World Health Organization
\end{abstract}

\section{Acknowledgements \\ The authors would like to acknowledge and thank the Systematic Review Services at the Discipline of Public Health, School of Nursing and Public Health in the College of Health Sciences, University of KwaZulu-Natal, for methodological guidance training and technical support.}

\section{Funding}

This review is not being funded. However, the full study for this protocol will be funded by the College of Health Sciences Scholarship, University of KwaZulu-Natal College of Health Sciences. The institution is also providing other resources and services in conducting this review.

\section{Availability of data and materials}

All data generated from this study will be included in the published scoping review article and will also be available on request.

\section{Authors' contributions}

MC conceptualised the study and prepared the manuscript under the guidance and supervision of TG and $\mathrm{KH}$. All authors contributed to the development and design of the study. TG and $\mathrm{KH}$ contributed to the methodology and reviewing of the manuscript. All authors contributed to the final version. All authors read and approved the final manuscript.

\section{Ethics approval and consent to participate \\ Not applicable.}

\section{Consent for publication}

Not applicable.

\section{Competing interests}

The authors declare no competing interests.

\section{Publisher's Note}

Springer Nature remains neutral with regard to jurisdictional claims in published maps and institutional affiliations.

Received: 30 January 2018 Accepted: 8 November 2018

Published online: 20 November 2018

\section{References}

1. Hubacher D, Trussell J. A definition of modern contraceptive methods. Contraception. 2015;92(5):420-1. Available from: http://www.sciencedirect. com/science/article/pii/S0010782415005430?via\%3Dihub. [cited 2018 Jan 18].

2. Nations U. Trends in Contraceptive Use Worldwide 2015. Department of Economic and Social Affairs PD, editor. 2015. Available from: http://www.un. org/en/development/desa/population/publications/pdf/family/ trendsContraceptiveUse2015Report.pdf. [cited 2018 Jan 18].

3. Nyarko SH. Prevalence and correlates of contraceptive use among female adolescents in Ghana. BMC Womens Health. 2015 [cited 2018 Jan 19];15(1): 60. Available from: https://doi.org/10.1186/s12905-015-0221-2.

4. McCurdy RJ, Schnatz PF, Weinbaum PJ, Zhu J. Contraceptive use in adolescents in sub-Saharan Africa: evidence from demographic and health surveys. Conn Med. 2014;78(5):261-72. Available from: http://www.ncbi.nlm. nih.gov/pubmed/24974559. [cited 2018 Jan 19]. 
5. Obare F, Birungi H, Undie C, Wanjiru M, Liambila W, Askew I. Levels, trends and determinants of contraceptive use among adolescent girls in Kenya. Nairobi: APHIA II OR Project in Kenya/ Population Council; 2011.

6. United Nations Population Fund. Universal access to reproductive health. Progress and challenges. 2016. Available from: https://www.unfpa.org/sites/ default/files/pub-pdf/UNFPA_Reproductive_Paper_20160120_online.pdf. [cited 2018 Jan 18].

7. Ministry of Health. Adolescent health strategic plan 2011 to 2015. 2015. Available from: http://www.un.org/en/development/desa/population/ publications/pdf/family/trendsContraceptiveUse2015Report.pdf. [cited 2018 Jan 18].

8. Curtis AC. Defining adolescence. J Adolesc Fam Heal. 2015;7(2):1-39. Available from: http://scholar.utc.edu/jafh/vol7/iss2/2. [cited 2018 Jan 18].

9. Adedokun O, Adeyemi O, Dauda C. Child marriage and maternal health risks among young mothers in Gombi, Adamawa state, Nigeria: implications for mortality, entitlements and freedoms. Afr Health Sci. 2016;16(4):986-99. Available from: http://www.ncbi.nlm.nih.gov/pubmed/28479891. [cited 2018 Jan 18]

10. Ganchimeg T, Ota E, Morisaki N, Laopaiboon M, Lumbiganon P, Zhang J, et al. Pregnancy and childbirth outcomes among adolescent mothers: a World Health Organization multicountry study. BJOG. 2014;121(s1):40-8. Available from: http://doi.wiley.com/10.1111/1471-0528.12630. [cited 2018 Jan 18]

11. Oyefara JL. Socio-economic consequences of adolescent childbearing in Osun State, Nigeria John Lekan Oyefara *. Kasbit Bus J. 2009;2(2):1-18. Available from: https://www.kasbit.edu.pk/KBJVol2/1-18.\%20SocioEconomic\%20Consequences\%20of\%20Adolescent\%20Childbearing\% 20in\%200sun\%20State,\%20Nigeria-John\%20Lekan\%200yefara.pdf. [cited 2018 Jan 18].

12. Branson N, Ardington C, Leibbrandt M. Health outcomes for children born to teen mothers in Cape Town, South Africa. Econ Dev Cult Change. 2015: 63(3):589-616. Available from: http://www.journals.uchicago.edu/doi/10. 1086/679737. [cited 2018 Jan 18].

13. Moher D, Stewart L, Shekelle P. Implementing PRISMA-P: recommendations for prospective authors. Syst Rev. 2016;5(1):15. Available from: http://www. systematicreviewsjournal.com/content/5/1/15. [cited 2018 Jan 26].

14. Arksey H, O'Malley L. Scoping studies: towards a methodological framework. Int J Soc Res Methodol. 2005;8(1):19-32. Available from: https://doi.org/10. 1080/1364557032000119616.

15. Levac D, Colquhoun H, O'Brien KK. Scoping studies: advancing the methodology. Implement Sci. 2010;5(1):69. Available from: http:// implementationscience.biomedcentral.com/articles/10.1186/1748-5908-5-69. [cited 2018 Jan 18].

16. The Joanna Briggs Institute. The Joanna Briggs Institute Reviewers' Manual 2015: Methodology for JBl scoping reviews. Joanne Briggs Inst. 2015;1-24. Available from: http://joannabriggs.org/assets/docs/sumari/Reviewers-Manual_ Methodology-for-JBI-Scoping-Reviews_2015_v1.pdf. [cited 2018 Jan 18].

17. Moher D, Liberati A, Tetzlaff J, Altman DG, Altman D, Antes G, et al. Preferred reporting items for systematic reviews and meta-analyses: the PRISMA statement. PLoS Med. 2009;6:e1000097. Available from: http://dx.plos.org/10. 1371/dssjournal.pmed.1000097. [cited 2018 Jan 19].

18. Castleberry A. NVivo 10 [software program]. Version 10. QSR International; 2012. Am J Pharm Educ. 2014;78(1).

19. Mheta D, Mashamba-Thompson TP. Barriers and facilitators of access to maternal services for women with disabilities: scoping review protocol. Syst Rev. 2017:6(1):99 Available from: https://doi.org/10.1186/s13643-017-0494-7.

20 Pluye P, Robert E, Cargo M, Bartlett G. Proposal: A mixed methods appraisal tool for systematic mixed studies reviews. Montréal McGill Univ. 2011;(Part I):1-8. Available from: http://mixedmethodsappraisaltoolpublic.pbworks. com/w/file/84371689/MMAT2011 criteriaandtutorial2011-06-29updated2014. 08.21.pdf. [cited 2018 Jan 17].

21. WHO. WHO | Adolescent health epidemiology. Who. World Health Organization; 2014. Available from: http://www.who.int/maternal_child_ adolescent/epidemiology/adolescence/en/. [cited 2018 Jan 18].

\section{Ready to submit your research? Choose BMC and benefit from:}

- fast, convenient online submission

- thorough peer review by experienced researchers in your field

- rapid publication on acceptance

- support for research data, including large and complex data types

- gold Open Access which fosters wider collaboration and increased citations

- maximum visibility for your research: over $100 \mathrm{M}$ website views per year

At BMC, research is always in progress.

Learn more biomedcentral.com/submissions 